\title{
IOT Based Building Monitoring System Using GSM Technique
}

\author{
C.Hemalatha ${ }^{1}$, M.Valan Rajkumar ${ }^{2}$, M.Gayathri ${ }^{3}$ \\ ${ }_{1,2}$ (Department of Electrical and Electrical Engineering, Gnanamani College of Technology, Namakkal, India)
}

\begin{abstract}
The Internet of Things (IoT) shall be able to incorporate transparently and seamlessly a large number of different and heterogeneous end systems, while providing open access to selected subsets of data for the development of digital services. Building a general architecture for the GSM IoT is hence a very complex task, mainly because of the extremely large variety of devices, link layer technologies, and services that may be involved in such a system. In this project mainly, focused to monitor the structural health of building and smart lighting in a particular area. The collected information of particular area is transfer to mobile devices through wireless media. In this paper, I focused specifically to an urban GSM IoT system that, while still being quite a broad category, are characterized by their specific application domain. Urban IoTs, in fact, are designed to support the Smart City vision, which aims at exploiting the most advanced communication technologies to support added-value services for the administration of the city and for the citizens.
\end{abstract}

Keywords: Constrained application protocol, Crack detection, Electrically Conductive Paint, Health Monitoring, RFID-tag, Sensor system integration.

\section{Introduction}

The Internet of Things (IoT) is a recent communication paradigm that envisions a near future, in which the objects of everyday life will be equipped with microcontrollers, transceivers for digital communication, and suitable protocol stacks that will make them able to communicate with one another and with the users, becoming an integral part of the Internet [1]. The IoT concept, hence, aims at making the Internet even more immersive and pervasive. Furthermore, by enabling easy access and interaction with a wide variety of devices such as, for instance, home appliances, surveillance cameras, monitoring sensors, actuators, displays, vehicles, and so on, the IoT will foster the development of a number of applications that make use of the potentially enormous amount and variety of data generated by such objects to provide new services to citizens, companies, and public administrations. This paradigm indeed finds application in many different domains, such as home automation, industrial automation, medical aids, mobile healthcare, elderly assistance, intelligent energy management and smart grids, automotive, traffic management, and many others [2-6].

If we have to reduce life cycle costs of a building from construction to maintenance, it is very effective to monitor structural health of a building and application on power electronics [7-11]. A damage identification system based on vibration measurements is effective for damage detection of whole structures or the story of a structure but it is not effective for damage detection of a specific portion of a building such as its structural members. On the other hand, damage detection based on phenomena such as cracking or heat is effective for damage detection of a specific portion of a building such as its structural members. By combining these two methods, it becomes possible to monitor structural health precisely [12-16].

A damage identification system based on vibration measurements has a possibility to find out the process of damage, but in such system, maintenance of sensor and data acquisition system costs very much. In some cases, it is not economical to measure for all time. It is very difficult to install such data acquisition system to many buildings because of high costs and application on power electronics with algorithms [17-25].

\section{Smart City Concept And Services}

The Smart City market has not really taken off yet, for a number of political, technical, and financial barriers. Under the political dimension, the primary obstacle is the attribution of decision-making power to the different stakeholders. A possible way to remove this roadblock is to institutionalize the entire decision and execution process, concentrating the strategic planning and management of the smart city aspects into a single, dedicated department in the city.

On the technical side, the most relevant issue consists in the non interoperability of the heterogeneous technologies currently used in city and urban development's. Finally, concerning the financial dimension, a clear business model is still lacking, although some initiative to fill this gap has been recently undertaken. The situation is worsened by the adverse global economic situation, which has determined a general shrinking of investments on public services. This situation prevents the potentially huge Smart City market from becoming reality. 
A possible way out of this impasse is to first develop those services that conjugate social utility with very clear return on investment, such as smart parking and smart buildings, and will hence act as catalyzes for the other added-value services.

\subsection{Structural Health of Buildings}

Proper maintenance of the historical buildings of a city requires the continuous monitoring of the actual conditions of each building and identification of the areas that are most subject to the impact of external agents. The urban IoT may provide a distributed database of building structural integrity measurements, collected by suitable sensors located in the buildings, such as vibration and deformation sensors to monitor the building stress, atmospheric agent sensors in the surrounding areas to monitor pollution levels, and temperature and humidity sensors to have a complete characterization of the environmental conditions. This database should reduce the need for expensive periodic structural testing by human operators and will allow targeted and proactive maintenance and restoration actions.

Finally, it will be possible to combine vibration and seismic readings in order to better study and understand the impact of light earthquakes on city buildings. This database can be made publicly accessible in order to make the citizens aware of the care taken in preserving the city historical heritage. The practical realization of this service, however, requires the installation of sensors in the buildings and surrounding areas and their interconnection to a control system, which may require an initial investment in order to create the needed infrastructure.

\subsection{Smart Lighting}

In order to support the 20-20-20 directive, the optimization of the street lighting efficiency is an important feature. In particular, this service can optimize the street lamp intensity according to the time of the day, the weather condition, and the presence of people. In order to properly work, such a service needs to include the street lights into the Smart City infrastructure. It is also possible to exploit the increased number of connected spots to provide WiFi connection to citizens. In addition, a fault detection system will be easily realized on top of the street light controllers.

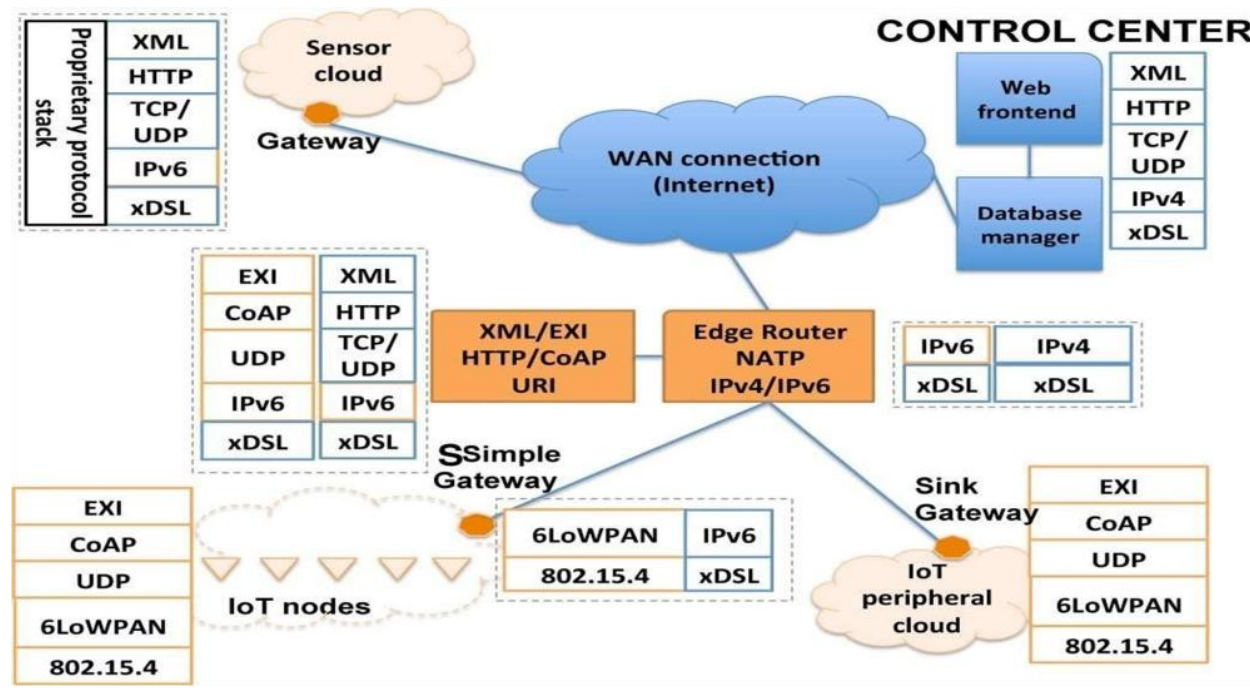

Figure 1. Components of IOT system

\subsection{Waste Management}

Waste management is a primary issue in many modern cities, due to both the cost of service and the problem is storage of garbage in landfills. A deeper penetration of ICT solution is used in this domain. However many result in significant saving and economical and ecological advantages. For instance, the use of intelligent waste containers, which detect be level of load and allow for an optimization of the collector trucks route, can reduce the cost of waste collection and improve the quality of recycling. To realize such a smart waste management service, the IOT shall connect the end devices.

\subsection{Urban IOT Architecture}

Smart City services are based on a centralized architecture, where a dense and heterogeneous set of peripheral devices deployed over the urban area generate different types of data that are then delivered through suitable communication technologies to a control center, where data storage and processing are performed. A primary characteristic of an urban IoT infrastructure is used. 
Hence, is its capability of integrating different technologies with the existing communication infrastructures in order to support a progressive evolution of the IoT, with the interconnection of other devices and the realization of novel functionalities and services. Another fundamental aspect is the necessity to make (part of) the data collected by the urban IoT easily accessible by authorities and citizens.

The point of departure is the definition which states that a city may be called 'smart' "when investments in human and social capital and traditional (transport) and modern (ICT) communication infrastructure fuel sustainable economic growth and a high quality of life, with a wise management of natural resources, through participatory government".

This holistic definition nicely balances different economic and social demands as well as the needs implied in urban development, while also encompassing peripheral and less developed cities. It also emphasizes the process of economic recovery for welfare and well-being purposes. Secondly, this characterization implicitly builds upon the role of the Internet and Web 2.0 as potential enablers of urban welfare creation through social participation, addressing hot societal challenges, such as energy efficiency, environment and health. The different components of an IOT system is shown in figure 1.

\subsection{Web Service Approach for IOT Service Architecture}

The practical implementation of a cleaning robot is an interdisciplinary problem but in this paper, the attention is focused on the actuators, sensors, and electronics and their usability. The mechanical design of a cleaning machine for domestic use must be ergonomic and small enough to move around the typical obstacles in a household room and as well as light enough for easy transportation in case of unexpected problems.

In its design, several different problems have to be solved: the efficiency of the cleaning device, the placement of the motorized wheels, the on-board sensors, and the battery recharge operation. The reference protocol architecture for IOT system is depicted in figure 2.

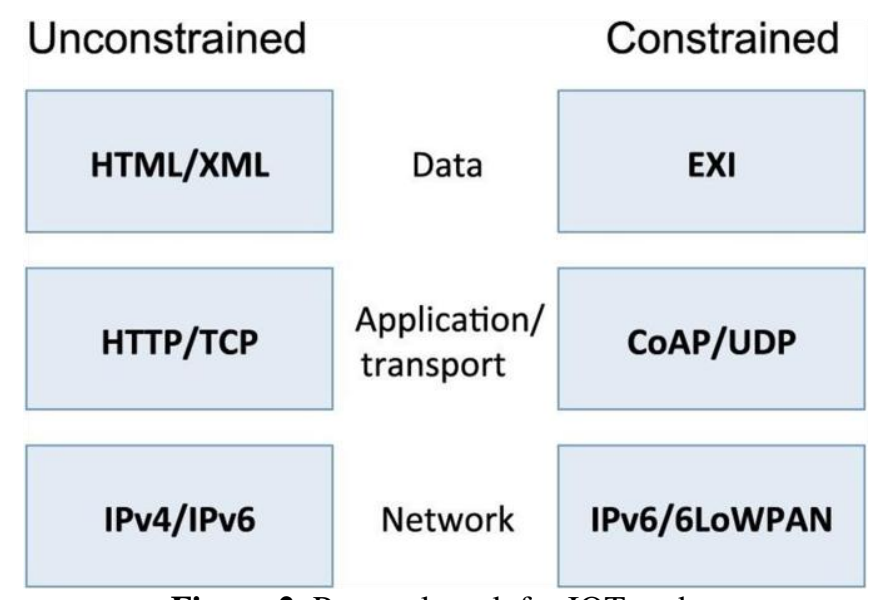

Figure 2. Protocol stack for IOT nodes

\subsection{Data Format}

As mentioned, the urban IoT paradigm sets specific requirements in terms of data accessibility. In architectures based on web services, data exchange is typically accompanied by a description of the transferred content by means of semantic representation languages, of which the Xtensible Markup Language (XML) is probably the most common. The size of XML messages is often too large for the limited capacity of typical devices for the IoT. Furthermore, the text nature of XML representation makes the parsing of messages by CPUlimited devices more complex compared to the binary formats. For these reasons, the working group of the World Wide Web Consortium (W3C) has proposed the EXI format, which makes it possible even for very constrained devices to natively support and generate messages using an open data format compatible with XML.

EXI defines two types of encoding, namely schema-less and schema-informed. While the schema-less encoding is generated directly from the XML data and can be decoded by any EXI entity without any prior knowledge about the data, the schema-informed encoding assumes that the two EXI processors share an XML Schema before actual encoding and decoding can take place. This shared schema makes it possible to assign numeric identifiers to the XML tags in the schema and build the EXI grammars upon such coding.

A general purpose schema-informed EXI processor can be easily integrated even in very constrained devices, enabling them to interpret EXI formats and, hence, making it possible to build multipurpose IoT nodes even out of very constrained devices. Using the schema-informed approach, however, requires additional care in the development of higher layer application, since developers need to define an XML Schema for the messages involved in the application and use EXI processors that support this operating mode. 
A generic framework for building IoT web applications according to the guidelines described in this section, the Asynchronous JavaScript and XML (AJAX) capabilities of modern web browsers that allow for a direct communication between the browser and the final IoT node, demonstrating the full internetworking of the protocol stack and the open data nature of the proposed approach.

\subsection{Application And Transport Layer}

Most of the traffic that crosses the Internet nowadays is carried at the application layer by HTTP over TCP. However, the verbosity and complexity of native HTTP make it unsuitable for a straight deployment on constrained IoT devices. For such an environment, in fact, the human-readable format of HTTP, which has been one of the reasons of its success in traditional networks, turns out to be a limiting factor due to the large amount of heavily correlated (and, hence, redundant) data. Moreover, HTTP typically relies upon the TCP transport protocol that, however, does not scale well on constrained devices, yielding poor performance for small data flows in loss environments.

The CoAP protocol overcomes these difficulties by proposing a binary format transported over UDP, handling only the retransmissions strictly required to provide a reliable service. Moreover, CoAP can easily interoperate with HTTP because: (i) it supports the ReST methods of HTTP (GET, PUT, POST, and DELETE), (ii) there is a one-to-one correspondence between the response codes of the two protocols, and (iii) the CoAP options can support a wide range of HTTP usage scenarios.

\subsection{Network Layer}

IPv4 is the leading addressing technology supported by Internet hosts. However, IANA, the international organization that assigns IP addresses at a global level, has recently announced the exhaustion of IPv4 address blocks. IoT networks, in turn, are expected to include billions of nodes, each of which shall be (in principle) uniquely addressable. A solution to this problem is offered by the IPv6 standard, which provides a 128-bit address field, thus making it possible to assign a unique IPv6 address to any possible node in the IoT network. While, on the one hand, the huge address space of IPv6 makes it possible to solve the addressing issues in IoT; on the other hand, it introduces overheads that are not compatible with the scarce capabilities of constrained nodes. This problem can be overcome by adopting 6LoWPAN, which is an established compression format for IPv6 and UDP headers over low-power constrained networks.

A border router, which is a device directly attached to the 6LoWPAN network, transparently performs the conversion between IPv6 and 6LoWPAN, translating any IPv6 packet intended for a node in the 6LoWPAN network into a packet with 6LoWPAN header compression format, and operating the inverse translation in the opposite direction.

While the deployment of a 6LoWPAN border router enables transparent interaction between IoT nodes and any IPv6 host in the Internet, the interaction with IPv4-only hosts remains an issue. More specifically, the problem consists in finding a way to address a specific IPv6 host using an IPv4 address and other meta-data available in the packet. In the following, we present different approaches to achieve this goal.

\section{Radio Frequency Identification (RFID)}

By the radio communication between RFID tag and transmitter with antenna and controller, the object can be recognized. Distance of this communication depends on the type of transmitter and tag. At the present time, there are RFID products whose communication distance is $2[\mathrm{~cm}]$ to $9[\mathrm{~m}]$. It is possible to communicate with a myriad tag by one transmitter because of the mobility of the transmitter. The price of tag itself is much more economical than conventional sensors.

\subsection{Crack Detection System}

Sensing part of this system shown in Figure 1 is a circuit in which electrically conductive material connects with copper wire part of the RFID tag. This sensing part is glued to detection objective part, and communication is carried out between transmitter and RFID tag. If the electrically conductive material is disconnected and the communication between RFID and transmitter is not possible, we can know the level of crack at the point electrically conductive material is applied. That is, the crack width is estimated by the ability of the electrically conductive material to conduct electricity. An example of coin type RFID tag is shown in Figure 3 


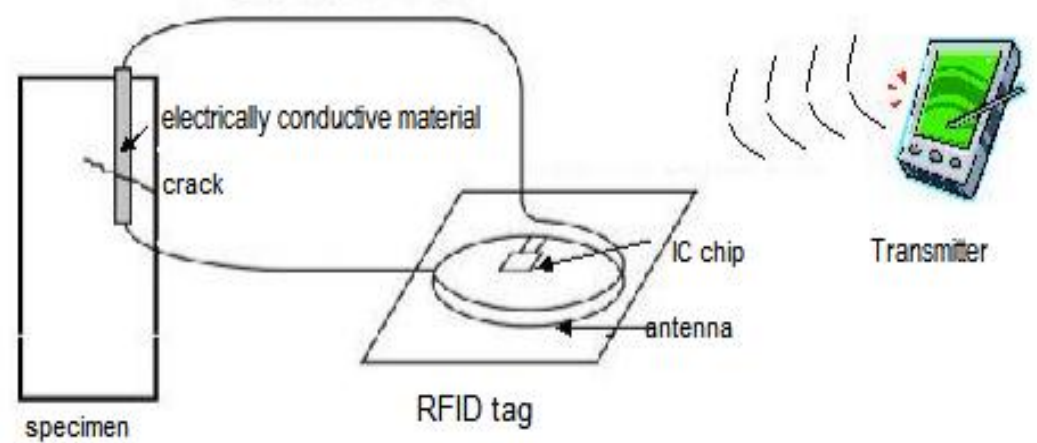

Figure 3. Outline of crack detection system

\subsection{Electrically Conductive Material}

What is necessary for electrically conductive material applied to the detection part is shown below:

- Electrically conductive material is disconnected at the crack width with the purpose.

- It is comparatively easy to apply.

- Material is economical.

In this study, two types of electrically conductive materials are tried. One is to apply paint directly which seem to be disconnected most easily, and the other is to glue a printed sheet which is suited to mass production. Both electrically conductive materials are very low cost, and the total installation cost with RFID-tag becomes very economical.

\subsection{Utilization of The Crack Detection Sensor}

In this crack detection sensor, the crack width is estimated from the line width of electrically conductive material that breaks. From results of experiments, there is some variation at wire breaking crack width. It is necessary to allow some variation in estimation of crack width from these results. For example, by using three types of line width electrically conductive materials, $2 \mathrm{~mm}, 0.2 \mathrm{~mm}$ and $0.6 \mathrm{~mm}$, prediction of crack level is possible such as small, medium or large size crack. Three types of level can be predicted from three types of electrically conductive materials.

It is possible to use this crack detection sensor for the health monitoring of piles in which visual inspection is very difficult. In such cases, the sensor is beforehand installed around at pile top or points of stratum changing in which cracks are predicted to occur. By the propriety of the communication with the RFID tag, the crack width is estimated.

On disconnection of electrically conductive material, it is necessary to prevent disconnection by the factor other than crack. About the printed sheet, it is possible to apply the laminate technology for protection of printed circuit board which is installed in products like cars or housing electric appliances. The coin type RFID tag is given in figure 4.

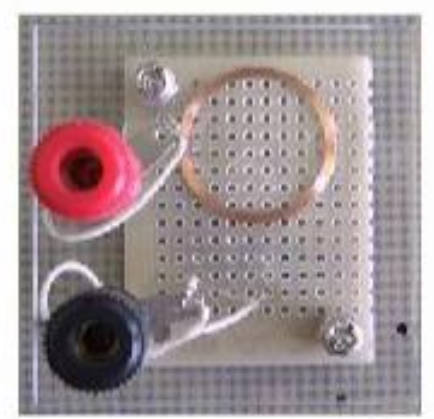

Figure 4. Coin type RFID tag

\section{Constrained Link Layer Technologies}

The IoT nodes mounted on the streetlight poles form a 6LoWPAN multihop cloud, using IEEE 802.15.4 constrained link layer technology. Routing functionalities are provided by the IPv6 Routing Protocol for Low power and Lossy Networks (RPL) [35]. IoT nodes are assigned unique IPv6 addresses, suitably compressed according to the 6LoWPAN standard. Each node can be individually accessible from anywhere in the Internet by means of IPv6/6LoWPAN. 
Nodes collectively deliver their data to a sink node, which represents the single point of contact for the external nodes. Alternatively, each node might publish its own features and data by running a CoAP server, though this feature is not yet implemented in the test bed. In either case, a gateway is required to bridge the 6LoWPAN cloud to the Internet and perform all the transcoding.

\subsection{WSN Gateway}

The gateway has the role of interfacing the constrained link layer technology used in the sensors cloud with traditional WAN technologies used to provide connectivity to the central backend servers. The gateway hence plays the role of 6LoWPAN border router and RPL root node. Furthermore, since sensor nodes do not support CoAP services, the gateway also operates as the sink node for the sensor cloud, collecting all the data that need to be exported to the backend services.

\subsection{HTTP-COAP PROXY}

The HTTP-CoAP proxy enables transparent communication with CoAP devices. The proxy logic can be extended to better support monitoring applications and limit the amount of traffic injected into the IoT peripheral network. For instance, it is possible to specify a list of resources that need to be monitored, so that the server can autonomously update the entries in a cache related to those devices. This mechanism can be supported by two different approaches:

1) by polling the selected resource proactively, thus enabling the implementation of traffic shaping techniques at the proxy or at the gateway and

2) by subscribing to the selected resource using the "observe" functionality of CoAP, thus enabling the server on the node to send the updates only when the value measured by the sensor falls outside a certain range. This service is co-located on the switchboard gateway in the Namakkal Smart City system, though it could also be placed in the backend servers, thus making it possible to control multiple gateways by using a single proxy instance.

\subsection{Database Server}

The database server collects the state of the resources that need to be monitored in time by communicating with the HTTP-CoAP proxy server, which in turn takes care of retrieving the required data from the proper source. The data stored in the database are accessible through traditional web programming technologies. The information can either be visualized in the form of a web site, or exported in any open data format using dynamic web programming languages.

\subsection{Operator Mobile Device}

Public lighting operators will be equipped with mobile devices that can locate the streetlight that requires intervention, issue actuation commands directly to the IoT node connected to the lamp, and signal the result of the intervention to the central system that can track every single lamppost and, hence, optimize the maintenance plan. Such a system can be successively extended to include other types of IoT nodes or clouds of IoT nodes, provided that each IoT peripheral system supports an HTTP-based interface, which makes it possible to interact with it in an open-, standard-, and technology-independent manner.

\section{Simulation And Result}

The discussed technologies are close to being standardized, and industry players are already active in the production of devices that take advantage of these technologies to enable the applications of interest.

This is the application where the simulations of the circuits can be tested. But the same file can be further processed to transform it into a layout. Layout is the final design which is needed in order to make the PCB of a circuit. The simulation diagram of smart city lighting is shown in figure 5. In fact, while the range of design options for IoT systems is rather wide, the set of open and standardized protocols is significantly smaller. The enabling technologies, furthermore, have reached a level of maturity that allows for the practical realization of IoT solutions and services, starting from field trials that will hopefully help clear the uncertainty that still prevents a massive adoption of the IoT paradigm. 


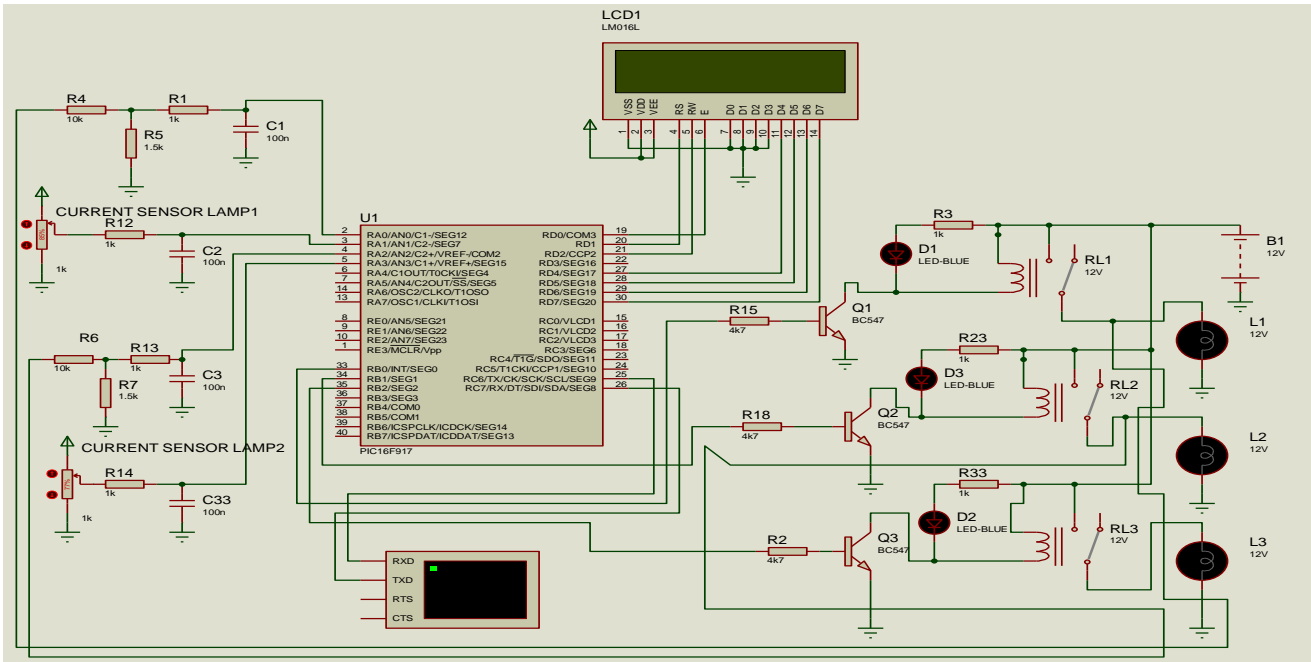

Figure 5. Simulation diagram of smart city lighting

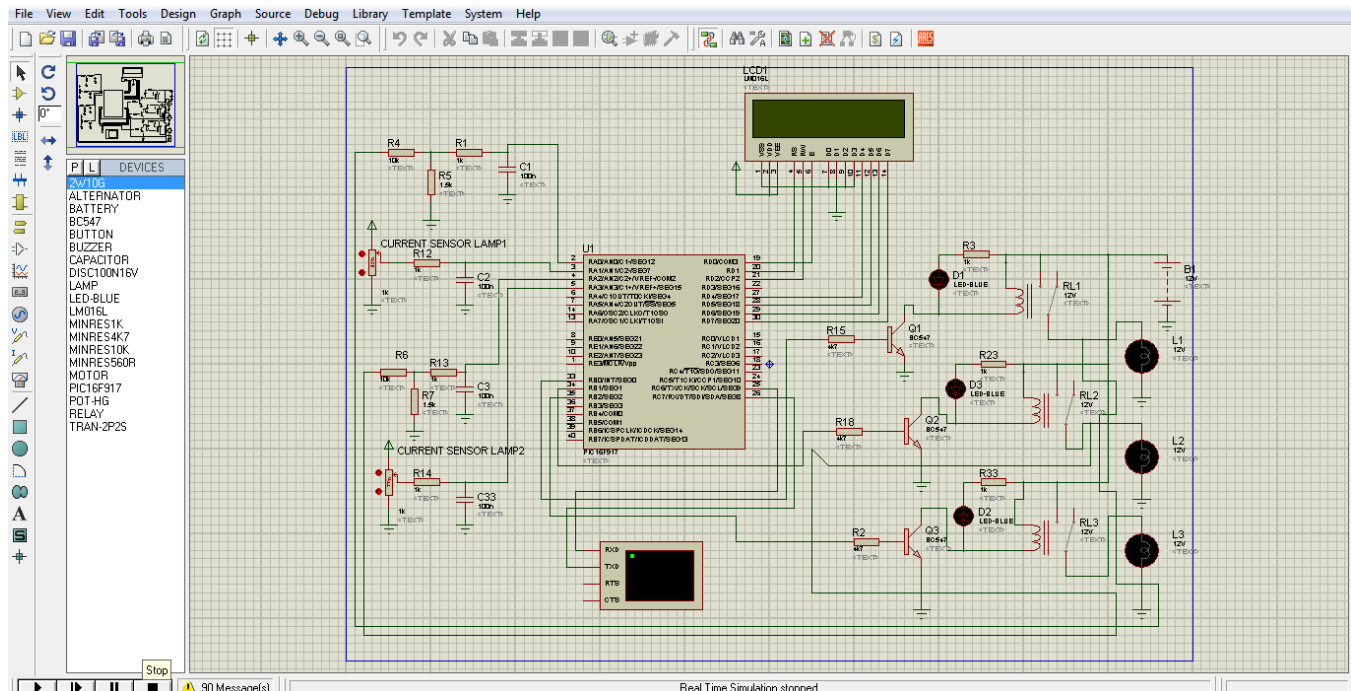

Figure 6. Proteus Simulation Window

To make the schematic, first we must have its raw design. Below is the schematic, that this documents uses to explain the steps to make the PCB. The simulation output for crack identification is shown in figure 7

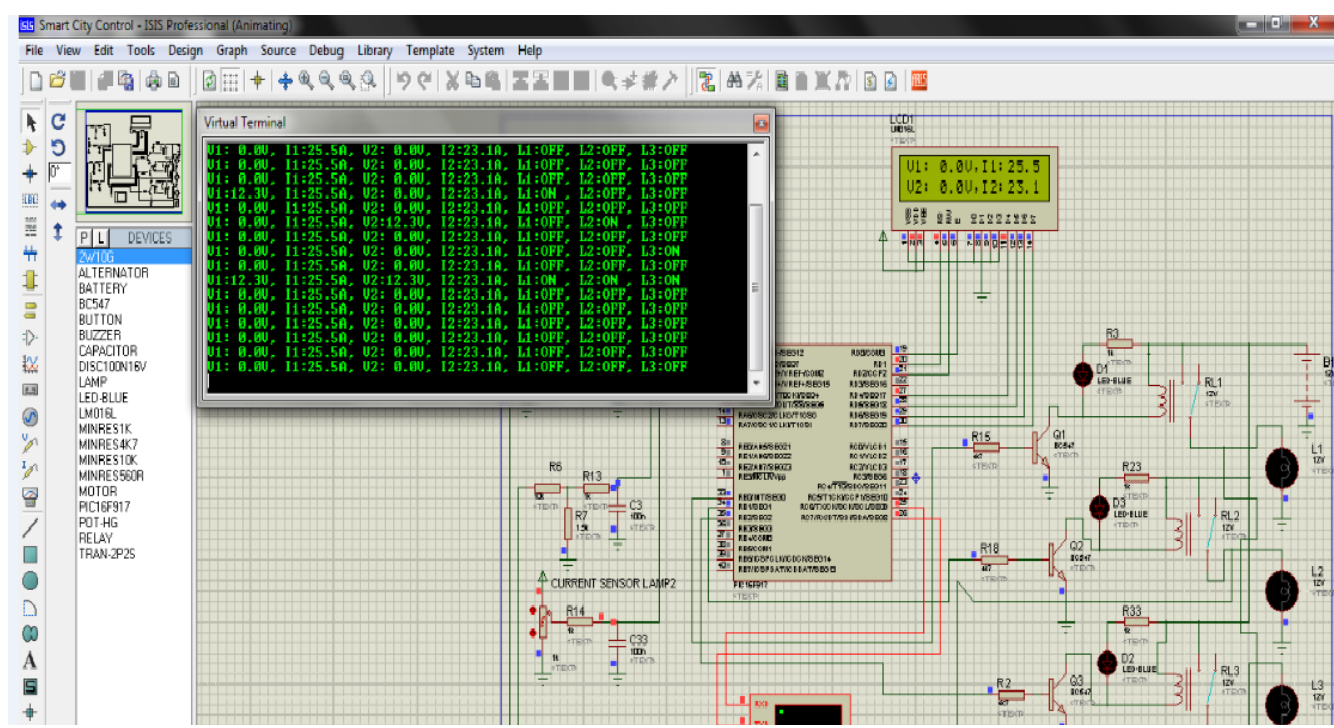

Figure 7. Simulation output for crack identification 


\section{Conclusion}

In this project, I analyzed the solutions currently available for the implementation of urban IoTs. The discussed technologies are close to being standardized, and industry players are already active in the production of devices that take advantage of these technologies to enable the applications of interest. In fact, while the range of design options for IoT systems is rather wide, the set of open and standardized protocols is significantly smaller. The enabling technologies, furthermore, have reached a level of maturity that allows for the practical realization of IoT solutions and services, starting from field trials that will hopefully help clear the uncertainty that still prevents a massive adoption of the IoT paradigm. The above mentioned project will be implemented in hardware in future phase 2 of the project work.

\section{References}

[1] L. Atzori, A. Iera, and G. Morabito,"The internet of things: A survey,"Comput. Netw, vol. 54, no. 15, pp. 2787-2805, 2010.

[2] N. Walravens and P. Ballon, "Platform business models for smart cities:From control and value to governance and public value,’IEEE Commun.Mag., vol. 51, no. 6, pp. 72-79, Jun. 2013

[3] M.Valan Rajkumar, P.S.Manoharan, Modeling and Simulation of Three-phase DCMLI using SVPWM for Photovoltaic System, Springer Lecture Notes in Electrical Engineering, under the volume titled "Power Electronics \& Renewable Energy Systems", Volume 326, Chapter No 5, January 2015, Pages 39-45.

[4] M.Valan Rajkumar, P.S.Manoharan, Harmonic Reduction of Fuzzy PI Controller based Three-Phase Seven-level DCMLI with SVPWM for Grid Connected Photovoltaic System, Journal International Review on Modeling and Simulations, Volume 6, No 3, June 2013, Pages 684-692.

[5] P.Thirumurugan, P.S.Manoharan, M.Valan Rajkumar, VLSI Based Space Vector Pulse Width Modulation Switching Control in the proceedings of IEEE International Conference on Advanced Communication Control and Computing Technologies ICACCCT 2012 on August 2012, ISBN No. 978-1-4673-2045-0 (Print) (Page):366-370.

[6] M.Valan Rajkumar, P.S.Manoharan, Space Vector Pulse Width Modulation of Three-Phase DCMLI with Neuro-Fuzzy MPPT for Photovoltaic System, World Journal of Modelling and Simulation, Volume 10, No 3, August 2014, Pages $193-205$.

[7] C. E. A. Mulligan and M. Olsson,"Architectural implications of smart city business models: An evolutionary perspective,"I EEE Commun. Mag vol. 51, no. 6, pp. 80-85, Jun. 2013

[8] A.Ravi, P.S.Manoharan, M.Valan Rajkumar, "Harmonic Reduction of Three-Phase Multilevel Inverter for Grid connected Photovoltaic System using Closed Loop Switching Control”, Journal-IREMOS, Volume 5, No 5, October 2012, Pages 1934-1942. ISSN: 1974-9821 (Print), 1974-982X (Online)

[9] P.Thirumurugan, P.S.Manoharan, M.Valan Rajkumar, "VLSI Based Inverter Switching Control" in the proceedings of International Conference on Mathematical Modeling and Applied Soft Computing MMASC'12 - Coimbatore Institute of Technology on July 2012, Vol-2 (Page):965-973.

[10] G.Vidhya Krishnan, M.Valan Rajkumar, C.Hemalatha, "Modeling and Simulation of 13-level Cascaded Hybrid Multilevel Inverter with less number of Switches", International Journal of Innovative Studies in Sciences and Engineering Technology, Volume 2, No 11, Nov.2016, Pages 43-47.

[11] M.Presser, L. A. Hernández Gómez, and J. Pettersson, "Smart Cities at the forefront of the future Internet," The Future Internet, Lect. Notes Comput. Sci., vol. 6656, pp. 447-462, 2011

[12] C. E. A. Mulligan and M. Olsson, "Architectural implications of smart city business models: An evolutionary perspective," IEEE Commun. Mag., vol. 51, no. 6, pp. 80-85, Jun. 2013.

[13] N. Walravens and P. Ballon, "Platform business models for smart cities: From control and value to governance and public value," IEEE Commun. Mag., vol. 51, no. 6, pp. 72-79, Jun. 2013.

[14] M.Valan Rajkumar, P.S.Manoharan, FPGA Based Multilevel Cascaded Inverters with SVPWM Algorithm for Photovoltaic system, Elsevier Journal Solar Energy, Volume 87, Issue 1, January 2013, Pages 229-245.

[15] C.Hemalatha, M.Valan Rajkumar, G.Vidhya Krishnan, "Simulation and Analysis for MPPT Control with Modified firefly algorithm for photovoltaic system”, International Journal of Innovative Studies in Sciences and Engineering Technology, Volume 2, No 11, Nov.2016, Pages 48-52.

[16] M.Valan Rajkumar, Prakasam, P. and Manoharan, P.S. (2016) Investigational Validation of PV Based DCDMLI Using Simplified SVM Algorithm Utilizing FPGA Tied with Independent Sources. Circuits and Systems, Volume 7, No 11, $3831-3848$. http://dx.doi.org/10.4236/cs.2016.711320

[17] M.Valan Rajkumar, P.S.Manoharan, "Modeling, Simulation and Harmonic Reduction of Three-Phase Multilevel Cascaded Inverters with SVPWM for Photovoltaic System”, Journal International Review on Modeling and Simulations, Volume 6, No. 2, April 2013, Pages 342-350. ISSN: 1974-9821 (Print), 1974-982X (Online)

[18] B. Sanjay Gandhi, S. Sam Chelladurai, and Dr. D. Senthil Kumaran, "Process Optimization for Biodiesel Synthesis from Jatropha Curcas Oil”, Taylor \& Francis-Distributed Generation and Alternative Energy Journal, Vol.23, No.4, Page 6- 16, 2011.

[19] M.Valan Rajkumar, P.S.Manoharan, "Modeling and Simulation of Five-level Five-phase Voltage Source Inverter for Photovoltaic Systems", Journal Przeglad Elektrotechniczny, Volume 10, No. 10, October 2013, Pages 237-241. ISSN: 0033-2097 (Print)

[20] B. Sanjay Gandhi and D. Senthil Kumaran, "The Production and Optimization of Biodiesel from Crude Jatropha Curcas Oil by a Two Step Process - An Indian Case Study Using Response Surface Methodology", Taylor \& Francis-International Journal of Green Energy, Vol.113, No.10, Page 1084-1096, 2014.

[21] S. Chandrasekar and Gian Carlo Montanari, "Analysis of Partial Discharge Characteristics of Natural Esters as Dielectric Fluid for Electric Power Apparatus Applications," IEEE Transactions on Dielectrics and Electrical Insulation, Vol. 21, No. 3, pp.1251-1259, June 2014.

[22] V.Jayaprakash Narayanan, B.Karthik and S.Chandrasekar,“ Flashover Prediction of Polymeric Insulators Using PD Signal TimeFrequency Analysis and BPA Neural Network Technique," Journal of Electrical Engineering and Technology. Vol. 9, Issue 4, pp. $1375-1384,2014$

[23] J. P. Lynch and J. L. Kenneth, “A summary review of wireless sensors and sensor networks for structural health monitoring," Shock and Vibration Digest, vol. 38, no. 2, pp. 91-130, 2006.

[24] T. Nuortio, J. Kytöjoki, H. Niska, and O. Bräysy, "Improved route planning and scheduling of waste collection and transport," Expert Syst. Appl., vol. 30, no. 2, pp. 223-232, Feb. 2006.

[25] A. R. Al-Ali, I. Zualkernan, and F. Aloul, "A mobile GPRS-sensors array for air pollution monitoring," IEEE Sensors J., vol. 10, no. 10, pp. 1666-1671, Oct. 2010. 